\title{
Transdermal rotigotine for the perioperative management of Parkinson's disease
}

\author{
Ullrich Wüllner • Jan Kassubek • Per Odin • \\ Michael Schwarz • Markus Naumann · Hermann-Josef Häck • \\ Babak Boroojerdi $\cdot$ Heinz Reichmann
}

Received: 15 February 2010/Accepted: 22 May 2010/Published online: 10 June 2010

(C) The Author(s) 2010. This article is published with open access at Springerlink.com

\begin{abstract}
Continuous delivery of antiparkinsonian medication during a perioperative period is desirable to avoid 'off'-symptom complications in surgical patients with concomitant Parkinson's disease (PD). Fourteen PD patients undergoing surgery under general anesthesia were switched from oral dopaminergic medication to transdermally delivered 24-h rotigotine (median dose $12 \mathrm{mg} / 24 \mathrm{~h}$ ) for the perioperative period. Rotigotine treatment was considered feasible by patients, their anesthesiologists and neurologists with good control of PD symptoms and easy switching and re-switching of PD medication.
\end{abstract}

For the NEUPOS Study Group.

U. Wüllner $(\bowtie)$

Department of Neurology, University Bonn,

Sigmund-Freud-Strasse 25, 53105 Bonn, Germany

e-mail: wuellner@uni-bonn.de

J. Kassubek

Department of Neurology, University of Ulm, Ulm, Germany

P. Odin

Department of Neurology, Hospital Bremerhaven-Reinkenheide,

Bremerhaven, Germany

M. Schwarz

Department of Neurology, Dortmund Hospital,

Dortmund, Germany

M. Naumann

Department of Neurology and Neurophysiology,

Central Hospital Augsburg, Augsburg, Germany

H.-J. Häck · B. Boroojerdi

UCB Pharma GmbH, Monheim, Germany

H. Reichmann

Department of Neurology, University of Technology,

Dresden, Germany
Keywords Parkinson's disease ·

Perioperative management $\cdot$ Rotigotine $\cdot$ Surgery ·

Transdermal/parenteral therapy

\section{Introduction}

Concomitant Parkinson's disease (PD) is a significant factor for perioperative morbidity in surgical patients (Mueller et al. 2009). Among other problems, cessation of oral dopaminergic medication during the perioperative period may result in a variety of 'off'-symptoms and increased peri and postoperative complications (GálvezJiménez and Lang 2004). Sudden withdrawal of dopaminergic medication may also cause parkinsonism-hyperpyrexia syndrome (Frucht 2004). Alternative routes of administration for continuous drug delivery such as nasogastric or intraduodenal levodopa, intravenous amantadine, or subcutaneous apomorphine are invasive and imply additional risks of ECG changes and nausea; intravenous amantadine is not available in the United States. Clinical data to support the safe perioperative use of these compounds are sparse. A retrospective analysis of data from two large multicenter trials suggested rotigotine, a non-ergot dopamine agonist with D3/D2/D1 activity, as a possible perioperative management option for PD patients (Korczyn et al. 2007). Rotigotine has been formulated for transdermal delivery in a 24-h patch, which ensures continuous drug release and stable plasma concentrations over a period of $24 \mathrm{~h}$ (Braun et al. 2005). This prospective, open-label, exploratory trial investigated the feasibility of switching PD patients scheduled for surgery under general anesthesia from their usual PD medication to rotigotine transdermal patch for the perioperative period. 
Table 1 Feasibility of switching to rotigotine treatment during the perioperative period rated by neurologists, anesthesiologists, and patients (full analysis set; $n=9$ )

\begin{tabular}{|c|c|c|c|c|c|c|}
\hline & $\begin{array}{l}\text { I completely agree } \\
1\end{array}$ & 2 & 3 & 4 & 5 & $\begin{array}{l}\text { I do not agree at all } \\
6\end{array}$ \\
\hline \multicolumn{7}{|l|}{ Neurologists } \\
\hline Switch from previous PD medication was easily feasible & $8(88.9 \%)$ & 0 & 0 & $1(11.1 \%)^{\mathrm{a}}$ & 0 & 0 \\
\hline Re-switch was easily feasible & $8(88.9 \%)$ & 0 & 0 & 0 & 0 & $1(11.1 \%)$ \\
\hline No unexpected PD symptoms perioperatively & $7(77.8 \%)$ & $2(22.2 \%)$ & 0 & 0 & 0 & 0 \\
\hline The patch is a feasible option & $8(88.9 \%)$ & $1(11.1 \%)$ & 0 & 0 & 0 & 0 \\
\hline \multicolumn{7}{|l|}{ Anesthesiologists } \\
\hline No unexpected PD symptoms perioperatively & $8(88.9 \%)$ & $1(11.1 \%)$ & 0 & 0 & 0 & 0 \\
\hline Handling of the patch was simple & $7(77.8 \%)$ & $2(22.2 \%)$ & 0 & 0 & 0 & 0 \\
\hline Handling was not time consuming & $8(88.9 \%)$ & $1(11.1 \%)$ & 0 & 0 & 0 & 0 \\
\hline The patch is a feasible option & $6(66.7 \%)$ & $2(22.2 \%)$ & 0 & $1(11.1 \%)^{\mathrm{a}}$ & 0 & 0 \\
\hline \multicolumn{7}{|l|}{ Patients } \\
\hline Perioperative patch treatment was easily feasible & $8(88.9 \%)$ & $1(11.1 \%)$ & 0 & 0 & 0 & 0 \\
\hline The symptoms of my PD were well controlled & $7(77.8 \%)$ & $2(22.2 \%)$ & 0 & 0 & 0 & 0 \\
\hline In the course of surgery, I felt safe with the PD patch & $6(66.7 \%)$ & $2(22.2 \%)$ & 0 & $1(11.1 \%)$ & 0 & 0 \\
\hline
\end{tabular}

Data are number of patients $(\%)$

$P D$ Parkinson's disease

a The same patient was rated ' 4 ' by the neurologist and the anesthesiologist

\section{Methods}

Patients and study design

Fourteen patients with idiopathic PD from six German trial sites were enrolled in this prospective, open-label, multicenter trial (NCT00594464) approved by the respective local institutional review boards and conducted according to the Declaration of Helsinki and Good Clinical Practice. Only patients who required PD medication, who were scheduled for an operation under general anesthesia, and who had a physical status classification according to the American Society of Anesthesiologists (ASA) (Camporesi et al. 1991) of stage II or III were included in the trial. All patients gave written informed consent.

Following a pretreatment visit (eligibility assessment) and baseline assessments on the day before surgery, patients received transdermal 24-h rotigotine patches at approximately 7 p.m. on the evening before surgery, replacing their regular PD medication(s) (defined as PD medication taken within 2 days prior to switching). The last administration of previous PD medication was at noon of the preoperative day for most PD medications, i.e., cabergoline was stopped upon baseline assessment and the last levodopa-containing preparations were administered in the evening of the pre-operative day. Rotigotine dose determination was at the discretion of the neurologist; general guidance regarding target doses was given according to published literature (LeWitt et al. 2007; Giladi et al. 2007; Poewe et al. 2007; Deutsche Gesellschaft für Neurologie website). After surgery, previous PD medication was to be resumed in the evening of the operative day; if required, rotigotine could be applied for up to 2 weeks following surgery. The trial was completed by a safety follow-up 2 weeks after discharge from the hospital.

\section{Clinical assessments}

Feasibility of switching to rotigotine treatment for the perioperative period was assessed by anesthesiologists on the day of surgery, and by neurologists and patients at safety follow-up using feasibility questionnaires (Table 1). Rating scales ranged from 1 (I completely agree) to 6 (I do not agree at all).

Safety [adverse events (AEs), vital signs, 12-lead ECG, clinical laboratory parameters] was monitored in all patients throughout the study. Additionally, blood samples for the determination of rotigotine plasma concentrations were obtained prior to the removal of the first 24-h patches and analyzed by liquid chromatography with tandem mass spectrometry. The apparent rotigotine dose is an estimate of the amount of rotigotine delivered to the skin within $24 \mathrm{~h}$ and is calculated as the difference of the initial drug content in the unused patch and the residual drug amount in the used patch. Rotigotine was quantified in the used first 24-h patches by a validated method.

The study design did not include collection of Unified Parkinson's Disease Rating Scale (UPDRS) data because it 
Table 2 Baseline characteristics of the study population $(n=14)$

\begin{tabular}{|c|c|c|c|c|c|c|c|c|}
\hline $\begin{array}{l}\text { Table } 2 \text { Baseline } \\
\text { characteristics of the study } \\
\text { population }(n=14)\end{array}$ & Patient & $\begin{array}{l}\text { Age } \\
\text { (years) }\end{array}$ & Gender & $\begin{array}{l}\text { BMI } \\
\left(\mathrm{kg} / \mathrm{m}^{2}\right)\end{array}$ & $\begin{array}{l}\text { Hoehn \& } \\
\text { Yahr stage }\end{array}$ & $\begin{array}{l}\text { PD } \\
\text { duration } \\
\text { (years) }\end{array}$ & $\begin{array}{l}\text { Substituted PD } \\
\text { medication } \\
\text { (mg levodopa } \\
\text { equivalent dose) }\end{array}$ & $\begin{array}{l}\text { Rotigotine } \\
\text { dose }(\mathrm{mg} / 24 \mathrm{~h})\end{array}$ \\
\hline & 10101 & 56 & Female & 25.8 & 2 & 3.1 & 905 & 16 \\
\hline & 10102 & 70 & Female & 25.7 & 3 & 7.0 & 500 & 16 \\
\hline & 10201 & 76 & Male & 23.0 & 2 & 6.7 & 370 & 8 \\
\hline & 10202 & 71 & Male & 27.3 & 2 & 2.7 & 400 & 8 \\
\hline & 10203 & 63 & Female & 29.1 & 1 & 11.9 & 1,400 & 14 \\
\hline & 10204 & 80 & Male & 30.9 & 3 & 9.3 & 800 & 6 \\
\hline & 10601 & 68 & Female & 25.0 & 3 & 14.7 & 775 & 8 \\
\hline & 10602 & 65 & Female & 27.3 & 1 & 2.7 & 670 & 12 \\
\hline & 10603 & 71 & Female & 30.5 & 3 & 20.7 & 100 & 2 \\
\hline & 10604 & 67 & Male & 28.7 & 4 & 12.8 & 1,295 & 16 \\
\hline & 10605 & 65 & Male & 24.9 & 4 & 8.0 & 700 & 16 \\
\hline & 10901 & 72 & Male & 24.0 & 3 & 0.6 & 150 & 6 \\
\hline & 11101 & 72 & Female & 24.1 & 1 & 10.2 & 650 & 12 \\
\hline$B M I$ body mass index, & 11201 & 66 & Male & 22.0 & 3 & 3.4 & 600 & 12 \\
\hline
\end{tabular}

$B M I$ body mass index, $P D$ Parkinson's disease

was felt that owing to the effect of surgery during the short study period, no clinically relevant statement about motor responses upon a change of PD medication was possible. An estimate is, however, provided by the answers of neurologists and patients to the feasibility questions ("unexpected symptoms of PD"). Owing to the exploratory character of the study, no formal sample size calculation was undertaken. Descriptive statistical analysis was performed with the SAS program (SAS Institute, Cary, NC, USA, version 9.1) using a full analysis set (FAS) patient population who had valid data for all three feasibility assessments. For a direct comparison of the substituted PD medications at doses of equivalent efficacy, all the dosages were converted to levodopa dosage equivalents according to the following formula (modified from Hobson et al. 2002):

Total levodopa equivalents $=$ regular levodopa dose $\times$ $1+$ levodopa continuous release dose $\times 0.75+([$ regular levodopa dose + continuous release levodopa dose $\times$ $0.75)] \times 0.25$ if taking tolcapone or entacapone) + pramipexole dose $\times 67+$ ropinirole dose $\times 16.67+$ pergolide dose $\times 100+$ bromocriptine dose $\times 10+$ cabergoline dose $\times 50+$ amantadine dose $\times 0.5+$ selegiline dose $\times$ $10+$ rasagiline dose $\times 100$.

Adverse events were encoded using the Medical Dictionary for Regulatory Activities version 9.1.

\section{Results}

Fourteen patients $(7$ female/7 male, mean age $68.7 \pm$ 5.9 years, mean Hoehn \& Yahr stage 2.5, mean PD duration $8.1 \pm 5.6$ years) received rotigotine treatment. Table 2 lists the baseline characteristics for each patient. Surgery was postponed in two patients; $12(85.7 \%)$ patients underwent surgery as per protocol, one of them withdrew his consent postoperatively.

Orthopedic surgery was the most common surgical procedure (7 patients); others included midfacial tumor extirpation, bladder extirpation, plastic surgery, pars plana vitrectomy and cerebral shunt implantation. Most patients (71.4\%) were on more than one PD medication within 2 days prior to switching. Substituted PD medication included levodopa (13), dopamine agonists (3 pramipexole, 3 ropinirole, 1 cabergoline), amantadine (4), catechol$O$-methyltransferase inhibitors (4), rasagiline (1) and biperiden (1). The mean levodopa equivalent dose for the substituted PD medication was $665 \pm 359 \mathrm{mg}$ (Table 2). The majority of patients $(64.3 \%)$ received rotigotine for $24 \mathrm{~h}$; three patients $(21.4 \%)$ were exposed for 2 days and two patients $(14.3 \%)$ for 5 days. The median rotigotine dose was $12 \mathrm{mg} / 24 \mathrm{~h}$ (range $2-16 \mathrm{mg} / 24 \mathrm{~h}$ ). Rotigotine doses for each patient are provided in Table 2 .

\section{Assessment outcomes}

All three feasibility assessments were available for nine patients $(64.3 \%)$ who were included in the FAS patient population. Table 1 lists the questionnaire ratings. The majority of neurologists (88.9\%), anesthesiologists (88.9\%) and all patients $(100 \%)$ completely agreed (rating of 1) or agreed (rating of 2) with the statement that rotigotine transdermal patch presents a feasible option for the perioperative management of PD patients. All patients stated that their PD symptoms were well controlled (rating of 1 or 2) which corresponded to the specialists' opinion that most 
patients did not show unexpected perioperative PD symptoms. Handling of the patch, switching and re-switching of PD medication were also rated easily feasible. In case of further surgery, the majority of the patients $(88.9 \%)$ would again choose rotigotine transdermal patch.

Ten patients $(71.4 \%)$ reported a total of 46 adverse events (AEs), mostly mild to moderate in intensity. At the end of the trial, $93 \%$ of AEs were listed as recovered; two AEs were recovered with sequelae (joint dislocation, dysesthesia) and one AE (iron deficiency) was ongoing. Five serious adverse events (SAEs) occurred; one was considered highly probably related to rotigotine treatment (hallucinations as described below), one may have been related (ventricular asystole as described below) and three were considered not drug related (mild post-procedural hematoma, moderate wound healing disturbance, and severe joint dislocation). A 72-year-old male on $6 \mathrm{mg} / 24 \mathrm{~h}$ rotigotine presented with moderate visual hallucinations (SAE) and severe nausea after surgery. The rotigotine dose remained unchanged and he recovered from the hallucinations on the same day and from nausea the day after. A 56-year-old female on $16 \mathrm{mg} / 24 \mathrm{~h}$ rotigotine had a ventricular asystole (SAE) after surgery which resolved spontaneously after $12 \mathrm{~s}$ without any therapeutic intervention. Rotigotine was withdrawn. The patient had experienced marked blood loss during surgery, requiring pharmacological intervention with catecholamines. Clinical chemistry assessed in the hours before the AE revealed low potassium and hemoglobin values; additionally, a low central venous pressure was reported. Concomitant medications at the time of the event were escitalopram oxalate (10 mg) once daily for depression and esomeprazole (40 mg) once daily for reflux oesophagitis. The patient had been on daily doses of $4.2 \mathrm{mg}$ pramipexole, $400 \mathrm{mg}$ of a levodopa/carbidopa/entacapone formulation and $100 \mathrm{mg}$ levodopa/carbidopa extended release 1/c which had been stopped the evening before surgery and resumed the day after surgery. Given the patient's clinical status during surgery and before the event, an association to rotigotine treatment seems unlikely but cannot be excluded.

There were no clinically relevant changes for the other safety parameters. Rotigotine plasma concentrations could be determined in ten patients $(71.4 \%)$ and ranged between 0.164 and $4.230 \mathrm{ng} / \mathrm{mL}$. Mean plasma concentrations (and apparent rotigotine doses) were in the same range as in previous clinical trials (Schwarz Pharma Ltd 2010; Güldenpfennig et al. 2005).

\section{Discussion}

Switching PD patients from oral dopaminergic medication to rotigotine transdermal patch for the perioperative period was considered feasible by a majority of neurologists, anesthesiologists, and their patients in this exploratory study. Handling of the patch was deemed simple, and switching and re-switching of PD medication easily feasible. Patient acceptance was high and, in case of further surgery, the majority would again choose rotigotine transdermal patch. Most patients had been on a regimen comprising at least two antiparkinsonian medications; apparently, previous polypharmacy did not influence the feasibility of switching to the patch.

Rotigotine absorption was not influenced by surgical procedures; apparent doses and plasma concentrations were within the published range (Schwarz Pharma Ltd 2010; Güldenpfennig et al. 2005).

Rotigotine-related adverse events were limited to two dopaminergic side effects in one patient (severe nausea and moderate hallucinations) and one ventricular asystole; and both were classified as serious. Both the patients recovered fully. While hallucinations and nausea are dopaminergic side effects, an association of the cardiac disorder to rotigotine seems unlikely. A recent thorough QT/QTc study in patients with advanced PD demonstrated that rotigotine application up to doses of $24 \mathrm{mg} / 24 \mathrm{~h}$ did not induce any QTc interval changes or other ECG abnormalities, indicating that the drug does not affect cardiac repolarization (Malik et al. 2008).

Considering the likely complications arising from discontinuation of oral dopaminergic PD medication with shorter half-lives during the perioperative period (Gálvez-Jiménez and Lang 2004; Frucht 2004) and the practical problems associated with current alternative parenteral administration routes, rotigotine transdermal patch may offer a feasible alternative for perioperative PD management.

Acknowledgments The authors would like to thank all participating patients, neurologists and anesthesiologists (H. Baas, F. Block, G. Deuschl, O. Kaut, B. Kutter, M. Löhle, S. Muhlack, G. Ochs, J.-P. Sieb, C. Sirtl, C. Schneider, F. Wappler, J. F. Zander). Further thanks go to E. Grosselindemann (Brett Medical Writing, Bibra Lake, Australia) and B. Brett (Brett Medical Writing, Pulheim, Germany) for editorial assistance and publication coordination. UCB Pharma S. A. undertook all costs associated with the development and the publishing of the present manuscript.

Conflict of interest statement The study was sponsored by UCB Pharma GmbH. UW served as consultant and lecturer and on advisory boards for Boehringer-Ingelheim, Glaxo-SmithKline, Lifescience KG, Novartis Pharmaceuticals, Orion Pharma, Schering AG, Schwarz-Pharma, Teva, UCB Pharma and received grant/research funding from BMBF, DFG, NAF, Stiftung Doppelfeld, Stiftung Verum, dPV, MerckKgaA, Pharmacia \& Upjohn. JK received honoraria for consultancy and lecturing from the sponsor and is a member of a local UCB advisory board. PO received consultancy fees from the sponsor. MS received lecture fees and is a member of a local UCB advisory board. $\mathrm{MN}$ has no conflict of interest. $\mathrm{HJH}$ and $\mathrm{BB}$ are employees of the study sponsor. HR received lecture fees and is part of a national and international UCB advisory board. 
Open Access This article is distributed under the terms of the Creative Commons Attribution Noncommercial License which permits any noncommercial use, distribution, and reproduction in any medium, provided the original author(s) and source are credited.

\section{References}

Braun M, Cawello W, Poole K, Horstmann R (2005) Steady-state pharmacokinetics of rotigotine in patients with early-stage Parkinson's disease. Eur J Neurol 12(Suppl 2):37-300 (abstract P 1245)

Camporesi EM, Greeley WJ, Lumb PD, Watkins WD (1991) Anesthesia. In: Sabiston DC Jr (ed) Textbook of surgery. WB Saunders, Philadelphia, pp 148-163

Deutsche Gesellschaft für Neurologie. Parkinson-Syndrome Leitlinien (Parkinson guidelines). Available at http://www.dgn.org/inhaltea-z/513-leitlinien-der-dgn-parkinson-syndrome-diagnostik-undtherapie.html (Accessed 7 May 2010)

Frucht SJ (2004) Movement disorder emergencies in the perioperative period. Neurol Clin N Am 22:379-387

Gálvez-Jiménez N, Lang AE (2004) The perioperative management of Parkinson's disease revisited. Neurol Clin N Am 22:367-377

Giladi N, Boroojerdi B, Korczyn AD, Burn DJ, Clarke CE, Schapira AHV (2007) Rotigotine transdermal patch in early Parkinson's disease: a randomized, double-blind, controlled study versus placebo and ropinirole. Mov Disord 22:2398-2404

Güldenpfennig WM, Poole KH, Sommerville KW, Boroojerdi B (2005) Safety, tolerability, and efficacy of continuous transdermal dopaminergic stimulation with rotigotine patch in early-stage idiopathic Parkinson disease. Clin Neuropharmacol 28:106-110

Hobson DE, Lang AE, Martin WR, Razmy A, Rivest J, Fleming J (2002) Excessive daytime sleepiness and sudden-onset sleep in Parkinson disease: a survey by the Canadian Movement Disorders Group. JAMA 287:455-463

Korczyn AD, Reichmann H, Boroojerdi B, Häck H-J (2007) Rotigotine transdermal system for perioperative administration. J Neural Transm 114:219-221

LeWitt PA, Boroojerdi B, MacMahon D, Patton J, Jankovic J (2007) Overnight switch from oral dopaminergic agonists to transdermal rotigotine patch in subjects with Parkinson disease. Clin Neuropharmacol 30:256-265

Malik M, Andreas J-O, Hnatkova K, Hoeckendorff J, Cawello W, Middle M, Horstmann R, Braun M (2008) Thorough QT/Qtc study in patients with advanced Parkinson's disease. Clin Pharmacol Ther 84:595-603

Mueller MC, Jüptner U, Wuellner U, Wirz S, Türler A, Hirner A, Standop J (2009) Parkinson's disease influences the perioperative risk profile in surgery. Langenbecks Arch Surg 394:511-515

Poewe WH, Rascol O, Quinn N, Tolosa E, Oertel WH, Martignoni E, Rupp M, Boroojerdi B (2007) Efficacy of pramipexole and transdermal rotigotine in advanced Parkinson's disease: a double-blind, double-dummy, randomised controlled trial. Lancet Neurol 6:513-520

Schwarz Pharma. Neupro ${ }^{\circledR}$ (Rotigotine Transdermal System). Available at http://www.ema.europa.eu/humandocs/PDFs/EPAR/neupro/ emea-combined-h626en.pdf (Accessed 7 May 2010) 